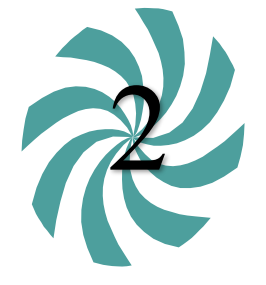

Tecnociencia, Vol. 22, $\mathrm{N}^{\circ} 2: 27-44$

julio-diciembre 2020

\title{
ON THE IDENTITY OF HOG-NOSED PIT-VIPERS FROM WESTERN PANAMA: A REVIEW OF SPECIMENS OF PORTHIDIUM LANSBERGII (SCHLEGEL, 1841) IN LOWER CENTRAL AMERICA.
}

\author{
${ }^{1,2,3}$ Claudio Manuel Monteza-Moreno, ${ }^{3,4}$ Carlos Ramos, ${ }^{5}$ Victor Martínez \\ \& ${ }^{6}$ Mahmood Sasa \\ ${ }^{1}$ Animal Behavior Graduate Group, University of California, Davis, Davis, CA. USA \\ ${ }^{2}$ Smithsonian Tropical Research Institute, Ancon, Panama, Republic of Panama \\ ${ }^{3}$ Estación Científica COIBA-AIP, Ciudad del Saber, Clayton, Panamá, República de \\ Panamá \\ ${ }^{4}$ Universidad de Panamá, República de Panamá, Facultad de Ciencias Naturales, \\ Exactas y Tecnología, Departamento de Genética y Biología Molecular \\ ${ }^{5}$ Universidad de Panamá, República de Panamá, Facultad de Ciencias Naturales, \\ Exactas y Tecnología, Centro para Investigaciones y Respuestas en Ofidiología \\ ${ }^{6}$ Universidad de Costa Rica, San José, Costa Rica, Facultad de Microbiología, Instituto \\ Clodomiro Picado \\ Corresponding author: e-mail: cmmonteza@ucdavis.edu; https://orcid.org/0000- \\ 0003-2537-2065 \\ Co-authors emails: laito52@yahoo.com (C. Ramos), pvmartinez@gmail.com (V. \\ Martínez), msasamarin@gmail.com, https://orcid.org/0000-0003-0118-5142 (M. \\ Sasa).
}

\begin{abstract}
Porthidium lansbergii is a relatively abundant pit viper in semiarid environments from eastern Panama, through the Caribbean plains and Magdalena Valley in Colombia to northeastern Venezuela. Like other members of this genus, P. lansbergii exhibits great variation in scutellation and other morphological characters, a situation that has complicated the taxonomy and identification of populations assigned to the species. In Lower Central America, P. lansbergii is known to inhabit seasonally-dry environments of the Pacific slopes of western Panama. Here, we aimed to clarify the taxonomic status of some enigmatic specimens collected in western Panama, with morphological
\end{abstract}


ambiguity, resembling $P$. lansbergii. We used the mitochondrial marker cytochrome oxidase $\mathrm{b}$ to perform a Bayesian phylogenetic analysis, to evaluate the affinities ofthese specimens with $P$. lansbergii individuals from central and eastern Panama, and Venezuela. We also analyzed meristic morphological characters to discriminate among populations of $P$. lansbergii in Panama, including other species of the Porthidium genus. Our analyses indicate that the enigmatic individuals do not differ from others individuals identified as $P$. lansbergii, thus forming a monophyletic group. We conclude that $P$. lansbergii has a more extended range than currently recognized including the western Pacific in Panama.

\section{KEY WORDS}

Porthidium lansbergii, Viperidae, morphometric characters, mitochondrial DNA, distribution, taxonomy.

\section{SOBRE LA IDENTIDAD DE LAS VÍBORAS DE NARIZ DE CERDO DEL OESTE DE PANAMÁ: UNA REVISIÓN DE ESPECÍMENES DE PORTHIDIUM LANSBERGII (SCHLEGEL, 1841) EN BAJA CENTROAMERICA}

\section{RESUMEN}

Porthidium lansbergii es una víbora relativamente abundante en ambientes semiáridos. Se distribuye desde el oeste panameño, hacia las llanuras del Caribe y el Valle de Magdalena en Colombia, hasta el noreste de Venezuela. Similar a otras especies del género, $P$. lansbergii, exhibe gran variación en su lepidosis y otros caracteres morfológicos. Esta situación taxonómica crea escenarios complejos para la identificación de ejemplares de esta especie. En Centro América, P. lansbergii es conocida por habitar ambientes estacionales, mayormente secos, en el este y sur de Panamá. Sin embargo, este trabajo procura aclarar el estado taxonómico de algunos especímenes enigmáticos colectados en el oeste de Panamá, con ambigüedad morfológica y que se asemejan a $P$. lansbergii. Utilizamos el marcador mitocondrial citocromo oxidasa $b$ para evaluar las afinidades de estos especímenes con individuos de P. lansbergii de centro y este de Panamá, así como de Venezuela, mediante análisis filogenético Bayesiano. Realizamos un análisis de caracteres morfométricos para discriminar entre poblaciones de P. lansbergii en Panamá, incluyendo otras especies del género. Nuestros resultados sugieren que los individuos enigmáticos no difieren de $P$. lansbergii, formando un grupo monofilético. Concluimos que la distribución de $P$. lansbergii incluye tierras del oeste pacifico panameño y es más amplia de lo que se reconoce actualmente. 


\section{PALABRAS CLAVES}

Porthidium lansbergii, Viperidae, caracteres morfométricos, ADN mitocondrial, distribución, taxonomía

\section{INTRODUCTION}

The genus Porthidium was described by Edward D. Cope (1871) to assign the nominal species $P$. nasutum and $P$. lansbergii, recognizing several anatomical features that separate them from other species then included in Bothrops (Bocourt, 1868) and Trigonocephalus (Schlegel 1841), respectively. As currently accepted, Porthidium includes nine small to medium sized pit-viper species that exhibit cryptic coloration and are often referred to as the hog-nosed pit-vipers due to their characteristic high rostral scale that tends to elevate-slightly or greatlythe tip of the snout (Campbell and Lamar, 2004; Köhler, 2003). Snakes in this genus are distributed at low to mid elevations in mesic and dry regions throughout most of Mesoamerica south to northwestern South America (Campbell and Lamar, 2004), comprising a monophyletic clade that has close affinities with the genera Cerrophidion and Metlapilcoatlus (Castoe et al., 2005; Campbell et al., 2019).

Most species within Porthidium are relatively abundant within their distributions, but the extent of variation in morphological and color pattern traits -and the great overlap of meristic characters among nominal species- have complicated proper identification throughout their range. Furthermore, early researchers created taxonomic confusion by including closely related Porthidium species into their description of nominal species (see Campbell and Lamar, 2004 for a review). Thus, it is not surprising that comprehensive morphological and/or molecular analyses of wide ranged species have revealed the existence of distinct populations that represent cryptic species, following their own evolutionary trajectory. For instance, $P$. porrasi, a hog-nosed pit-viper that inhabits lowland mesic forest in the southern Pacific of Costa Rica (Lamar and Sasa, 2003), was previously recognized as a distinct population of P. nasutum (Porras et al., 1981; Savage, 2002).

In the case of $P$. lansbergii (type-locality: Turbaco, Colombia), the 
problem has been even more complicated. For this species, several subspecies have been previously proposed, and it was suspected that $P$. lansbergii may be polytypic (Campbell and Lamar 1989, 2004). The distributional range of $P$. lansbergii includes the Caribbean plains and the Magdalena Valley in northern Colombia eastward to northern and central Venezuela, including the Maracaibo basin, ranging in elevation from sea level to at least $1270 \mathrm{~m}$ (Campbell and Lamar, 2004). The species also occurs in coastal plains of the Pacific from eastern (Darien region) to central Panama (Fig 1.). Along its range, P. lansbergii occurs mainly in seasonally dry environments, although it also inhabits humid forests of eastern Panama and Colombia (Campbell and Lamar, 2004).

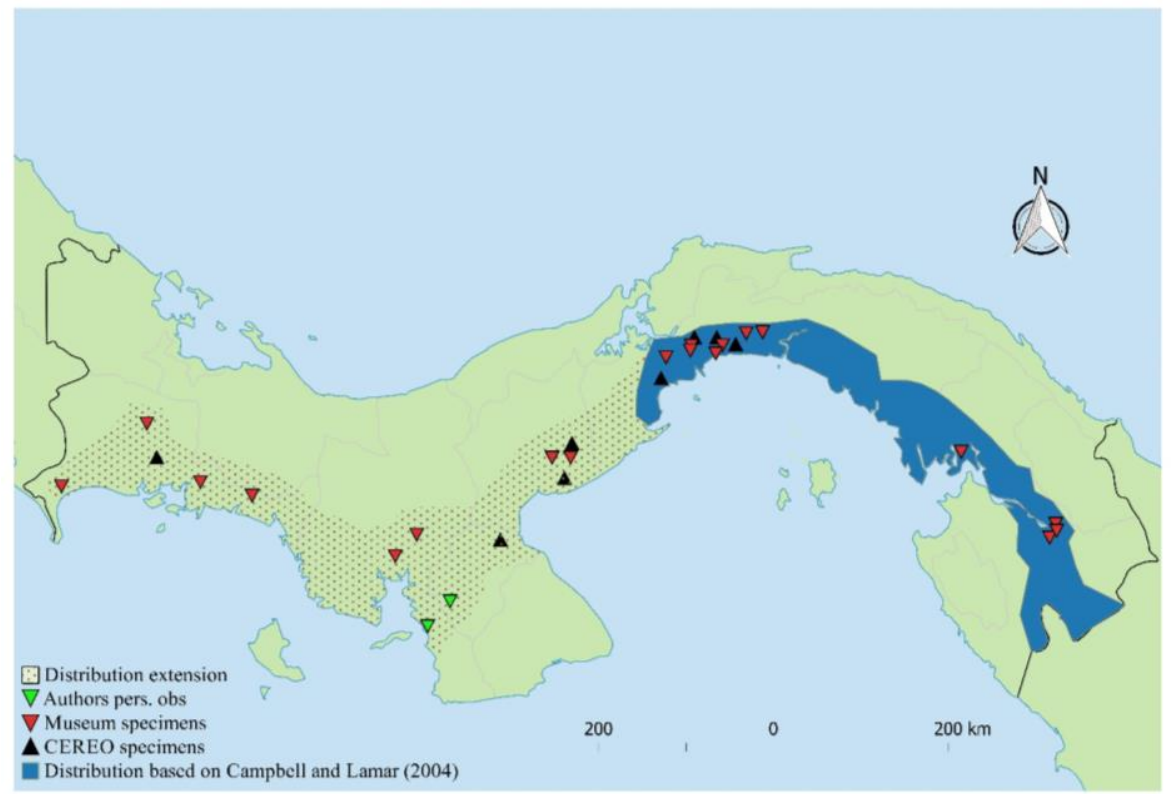

Fig. 1 Distribution of $P$. lansbergii in Panama. Map of Panama showing the distribution based on Campbell and Lamar (2004) in blue; specimens from the CEREO collection in black triangles, from museums in red triangles, and observed by authors in green triangles. Point patterns represents the extended distribution for $P$. lansbergii presented herein

Although $P$. lansbergii has been extensively collected in central and eastern Panama, its status in western Panama remains unclear. A few 
museum records account for collection localities scattered throughout the Pacific coast on the occidental provinces of Chiriqui, Veraguas, and Cocle. For instance, H. Clark recorded an individual in 1936 from Puerto Armuelles, in Chiriqui Province (MCZ:Herp:R-42731, Fig. 2), which represents a range extension over $350 \mathrm{~km}$ from known localities in central Panama. Moreover, several enigmatic individuals, with ambiguous morphology, were collected at some localities in the western Pacific of Panama (Fig. 3). These specimens were housed at the snake collection in the 'Centro para Investigaciones y Respuestas en Ofidiología (CEREO), Facultad de Ciencias Naturales, Exactas y Tecnologia' at the University of Panama. Based only in the folidosis, these specimens were, tentatively, identified as $P$. cf. lansbergii by Martinez, V. However, the atypical coloration patterns observed (V. Martinez, pers. obs.) in these individuals made their taxonomy confusing (Fig. 3). Therefore, we aimed to clarify these enigmatic individuals' identification. We compared morphometric characters, sequenced mitochondrial DNA fragment cytochrome oxidase $b$ and conducted an intensive literature search. We formally assigned them to $P$. lansbergii, thus providing strong evidence to support that its distributional range should be extended to the western Pacific slope of Panama.

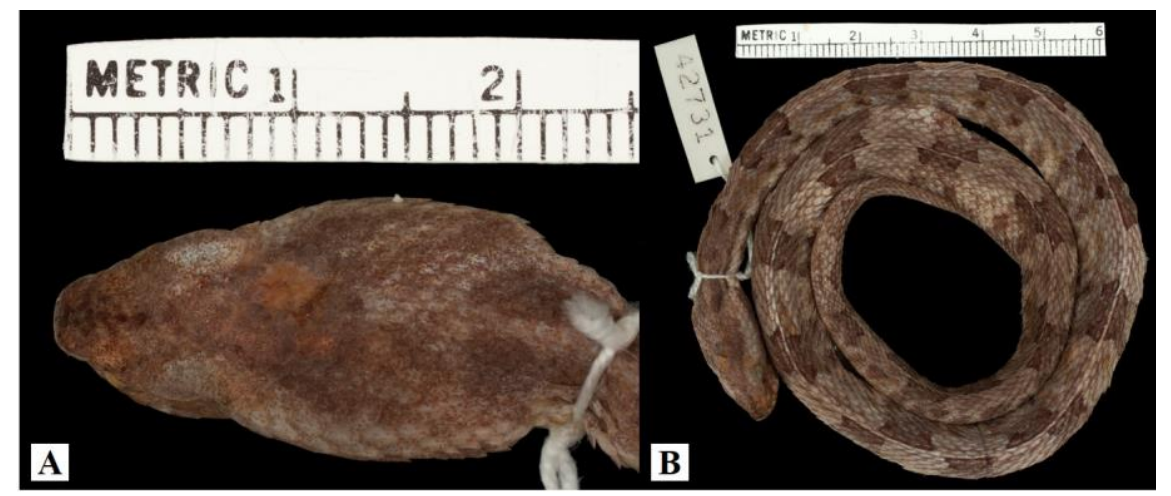

Fig. 2 Specimen collected by H. Clark in Puerto Armuelles, Chiriqui (western Panama), with museum record MCZ:Herp:R-42731 

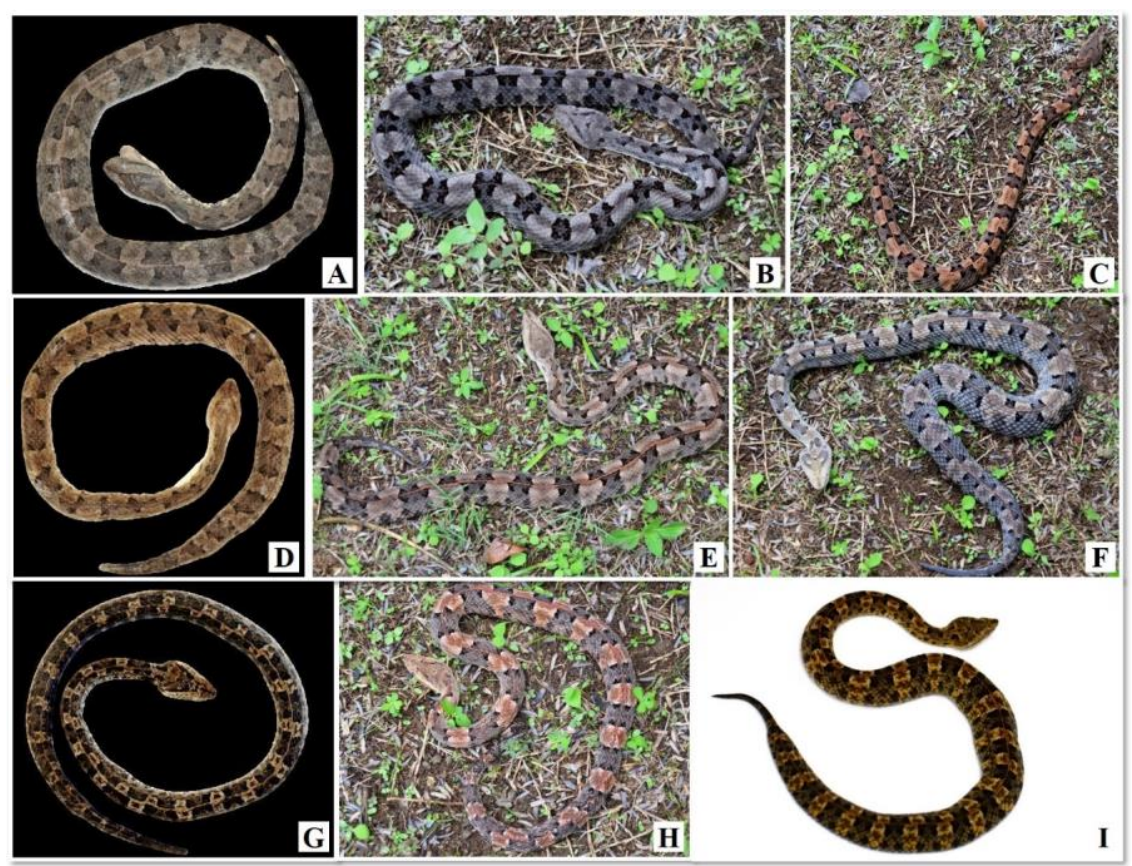

Fig. 3 P. lansbergii specimens collected from central Panama (figs. 3B, $\mathrm{C}, \mathrm{D}, \mathrm{G}, \mathrm{H}$ ) and western Panama (figs. 3A, E, F, I)

\section{METHODS}

We assessed the morphological traits that discriminate between Porthidium lansbergii from Panama and related species within the genus. We performed morphological analysis on 9 enigmatic $P$. cf lansbergii individuals held at the CEREO and compared them to 114 Porthidium specimens kept in the following institutions: California Academy of Science (CAS), Field Museum of Natural History, Chicago, IL (FMNH), Florida Museum of Natural History (FLMNH), University of Kansas Natural History Museum and Biodiversity Center (KU), Museum of Vertebrate Zoology, University of California, Berkeley (MVZ), Museo de Zoologia, Universidad de Costa Rica (MZUCR), and the National Museum of Natural History (NMNH).

In total, we included 4 P. lansbergii specimens from eastern Panama, 15 
from central Panama, and $9 P$. cf lansbergii from western Panama (see Table 1 for specimens' localities); 39 P. ophryomegas, 3 P. volcanicum, $3 P$. porrasi, and 50 P. nasutum (see Table 2 for meristic data). For each specimen, we identified sex and counted the number of body blotches, as well as the number of the following scales: dorsal (at head, mid-body, and anal region), ventral, caudal, supralabial, infralabial, canthal and internasal. We also measured total body length and tail length (to the nearest $0.1 \mathrm{~cm}$ ). Sexual dimorphism in meristic characters was evaluated using parametric mean comparisons. We applied Principal Component Analysis (PCA) to the correlation matrix to determine the existence of patterns in scale meristic characters among populations of all Porthidium species included, as well as among specimens of $P$. cf lansbergii and $P$. lansbergii to distinguish subpopulations. We implemented all statistical analyses in JMP Pro $13 \AA$ (SAS Institute Inc, 1989-2019).

We evaluated phylogenetic relationships between P. cf. lansbergii and other members of the group. We collected blood samples from 7 P. $c f$. lansbergii individuals located at the CEREO, and extracted DNA using the DNeasy ${ }^{\circledR}$ Blood \& Tissue kits following the manufacturer's protocol (QIAGEN, 2006). We used the primers Gludg (5'TGACTTGAARAACCA YCGTTG-3') and Atrcb3 (5'TGAGAAGTTTTC YGGGTCRT T-3') (Palumbi, 1996). We adjusted the protocol described by Chippindale et al (2000) and amplified the subunit $c y t-b$ in a Perkin-Elmer 2400 thermocycler under the following conditions: 1 minute at $95^{\circ} \mathrm{C}$ and 15 seconds at $94^{\circ} \mathrm{C}$, one minute at $54^{\circ} \mathrm{C}$, and one minute at $72^{\circ} \mathrm{C}$ with a final extension of 7 minutes at $72^{\circ} \mathrm{C}$. We purified the PCR products using MinElute reaction cleanup kit (QIAGEN) and then sequenced using BigDye ${ }^{\circledR}$ technology (PE Applied Biosystems) in an ABI PRISM® 310 sequencer. All Porthidium lansbergii sequences were deposited in GenBank with the following accession number: MN597002 (CEREO109), MN597003 (CEREO115), MN597004 (CEREO116), MN597005 (CEREO118), MN597006 (CEREO120), MN597007 (CEREO213), MN597008 (CEREO235). The phylogenetic analysis was performed under Metropolis-Hastings coupled Markov Chain Monte Carlo Bayesian methods (BMCMC) using the program MEGAS 5.10 and results were 
visualized with TreeView. In addition to our Panamanian samples (sequences for $P$. lansbergii specimens are available per request to the corresponding author), we included the following Porthidium sequences as ingroups or near outgroups (GenBank ${ }^{\circledR}$ accession numbers): $P$. lansbergii (DQ061206, central Panama) and (AY223582, Venezuela); $P$. arcosae (AF292575); P. dunni (DQ061218); P. nasutum (AY223579); P. ophryomegas (DQ061241, plus two individuals from Costa Rica housed at Instituto Clodomiro Picado); $P$. porrasi (DQ061211); and $P$. yucatanicum (DQ061215). In addition, sequences from Metlapilcoatlus nummifer (=mexicanus; AY223584), Atropoides. picadoi (DQ061197), Cerrophidion godmani (AY223578), C. petlalcalensis (DQ061202.1), and C. tzotzilorum (DQ061204) were assigned as outgroups.

Table 1. Information of collection for P. lansbergii specimens from Panamá

\begin{tabular}{|c|c|c|c|c|}
\hline Voucher & Region & Locality & Year & Collector \\
\hline CEREO-120 & Central & Las Pavas, Arraijan, Panama Oeste province & NA & NA \\
\hline CEREO-213 & Central & Tocumen, Panama province & NA & NA \\
\hline CAS-21061 & Central & Canal Zone, vic. Fort Clayton, Panama province & 1958 & "H.M. Smith" \\
\hline CAS-21062 & Central & Canal Zone, vic. Fort Clayton, Panama province & 1958 & "H.M. Smith" \\
\hline MVZ-78766 & Central & Chepo $(9.16667,-79.1)$, Panama province & 1964 & N. Gale \\
\hline FLMNH-39818 & Central & NA & 1968 & Sam R. Telford \\
\hline FLMNH-74277 & Central & Canal Zone, Panama province & 1967 & D. Bruce Means \\
\hline FMNH-Chi-68051 & Central & NA & NA & NA \\
\hline CEREO-112 & Central & San Juan de Pequeni, Pacora, Panama province & NA & NA \\
\hline CEREO-116 & Central & NA & NA & NA \\
\hline CEREO-118 & Central & El Palmar, Panama province & NA & NA \\
\hline CAS-98362 & Central & $\begin{array}{l}\text { Tocumen Airport (8.18333, -77.68333), Panama } \\
\text { province }\end{array}$ & 1964 & "T.J. Papenfuss" \\
\hline FLMNH-39819 & Central & NA & 1968 & Sam R. Telford \\
\hline KU-112588 & Central & Juan Diaz, Panama province & 1961 & NA \\
\hline KU-112576 & Central & $\begin{array}{l}28 \text { km ENE Panama City, Rio Pacora, Panama } \\
\text { province }\end{array}$ & 1965 & NA \\
\hline KU-112579 & Eastern & Rio Tuira at Rio Mono, Darien province & 1965 & NA \\
\hline NMNH-140676 & Eastern & El Real de Santa Maria, Darien province & 1958 & J. Hardy \\
\hline MVZ-83442 & Eastern & Yaviza, Darien province & 1967 & C. M. Cavalier \\
\hline KU-112578 & Eastern & 3 km E El Real, Rio Tuira, Darien province & 1965 & NA \\
\hline CEREO-109 & Western & Santiago, Veraguas province & NA & NA \\
\hline CEREO-115 & Western & Puerto Frio, Penonome, Cocle province & NA & NA \\
\hline CEREO-235 & Western & Valle de Anton, Cocle province & NA & NA \\
\hline CEREO-329 & Western & Las Lomas, Boquete, Chiriqui province & NA & NA \\
\hline NMNH-129876 & Western & Mojarras, Veraguas province & 1951 & M. Stirling \\
\hline NMNH-297735 & Western & $\begin{array}{l}\text { Cerro Colorado, Escopeta Camp, ca. } 23 \mathrm{~km} \mathrm{NE} \\
\text { of San Felix, Chiriqui province }\end{array}$ & $\begin{array}{l}1969- \\
1980 .\end{array}$ & A. Forero \\
\hline FLMNH-127697 & Western & NA & 1970 & Nicholas Nell \\
\hline KU-112580 & Western & $5 \mathrm{~km}$ NW San Lorenzo, Chiriqui province & 1966 & NA \\
\hline
\end{tabular}


Table 2. Data for meristic characters for the five Porthidium species included in this study. Species $1=P$. nasutum, $2=P$. ophryomegas, $3=P$. lansbergii, $4=P$. volcanicum, $5=P$. porrasi; (1) ventral scales, (2) caudal scales, (3) head dorsal scales, (4), mid-dorsal scale (5) anal dorsal scale row, (6) supralabial scales, (7) infralabial scales, (8) canthal scale, (9) internasal scale, $(10)$ gender $(0=$ female, $1=$ male $),(11)$ body blotches, (12) total body length (cm), and (13) tail length

\begin{tabular}{|c|c|c|c|c|c|c|c|c|c|c|c|c|c|c|}
\hline Museum Catalog & \begin{tabular}{|l|} 
Species \\
\end{tabular} & 1 & 2 & 3 & 4 & 5 & 6 & 7 & 8 & 9 & 10 & 11 & 12 & 13 \\
\hline MZUCR-109 & 1 & 143 & 28 & 25 & 23 & 19 & 10 & 12 & $1 \mid$ & 6 & 1 & 30 & 51.5 & 4.9 \\
\hline MZUCR-110 & 1 & 139 & 27 & 25 & 23 & 19 & 10 & 12 & $1 \mid$ & 5 & 1 & 21 & 58.1 & 6 \\
\hline MZUCR-162 & 1 & 138 & 28 & 23 & 23 & 19 & 10 & 12 & 1 & 5 & 1 & 22 & 44.2 & 4.5 \\
\hline MZUCR-1870 & 1 & 130 & 27 & 23 & 23 & 19 & 9 & 11 & 1 . & 6 & 1 & 18 & 26 & 3.6 \\
\hline MZUCR-2721 & 1 & 136 & 31 & 23 & 23 & 19 & 8 & 10 & 1 & 6 & 1 & 28 & 34.9 & 5.5 \\
\hline MZUCR-2739 & 1 & 139 & 34 & 23 & 23 & 23 & 9 & 11 & $1 \mid$ & 4 & 1 & 25 & \begin{tabular}{|l|}
31.4 \\
\end{tabular} & 5 \\
\hline MZUCR-2941 & 1 & 144 & 32 & 25 & 23 & 19 & 10 & 12 & 1 & 6 & 1 & 25 & 56.5 & 6.5 \\
\hline MZUCR-4647 & 1 & 139 & 28 & 23 & 23 & 19 & 9 & 11 & \begin{tabular}{|l|l|}
1 \\
\end{tabular} & 5 & 1 & 24 & 21.6 & 1.8 \\
\hline MZUCR-5743 & 1 & 145 & 27 & 25 & 23 & 19 & 10 & 12 & 1 & 4 & 1 & 24 & \begin{tabular}{|l|}
41.7 \\
\end{tabular} & 4 \\
\hline \begin{tabular}{|l|} 
MZUCR-5998 \\
\end{tabular} & 1 & 144 & 30 & 27 & 25 & 21 & 10 & 12 & 11 & 5 & 1 & 18 & 25.5 & 3.2 \\
\hline MZUCR-6767 & 1 & 137 & 28 & 25 & 23 & 19 & 10 & 12 & \begin{tabular}{|l|}
1 \\
\end{tabular} & 5 & 1 & 21 & \begin{tabular}{|l|}
34.1 \\
\end{tabular} & 4.5 \\
\hline MZUCR-7238 & 1 & 139 & 27 & 23 & 23 & 19 & 10 & 12 & $1 \mid$ & 5 & 1 & 22 & 48 & 5.6 \\
\hline MZUCR-10230 & 1 & 138 & 27 & 25 & 23 & 21 & 9 & 11 & $1 \mid$ & 5 & 1 & 26 & 30.5 & 4.2 \\
\hline MZUCR-10304 & 1 & 143 & 31 & 23 & 23 & 21 & 9 & 11 & 1 & 5 & 1 & 34 & 32.5 & 4.8 \\
\hline MZUCR-10305 & 1 & 127 & 27 & 25 & 25 & 21 & 10 & 12 & 1 & 5 & 1 & 27 & 64.6 & 6.6 \\
\hline MZUCR-10362 & 1 & 131 & 27 & 25 & 25 & 21 & 10 & 12 & 1 & 5 & 1 & 19 & 25.1 & 3 \\
\hline MZUCR-10413 & 1 & 132 & 26 & 25 & 25 & 19 & 9 & 11 & 1 & 5 & 1 & 22 & 34.4 & 4.1 \\
\hline MZUCR-11150 & 1 & 135 & 28 & 23 & 23 & 19 & 10 & 12 & 1 . & 5 & 1 & 27 & 49.8 & 5.7 \\
\hline MZUCR-12612 & 1 & 144 & 27 & 25 & 23 & 19 & 10 & 12 & 1 & 5 & 1 & 25 & 53 & 5.9 \\
\hline MZUCR-12809 & 1 & 141 & 23 & 27 & 25 & 21 & 11 & 13 & 1 & 5 & 1 & 20 & 58.4 & 5.5 \\
\hline MZUCR-13800 & 1 & 143 & 27 & 25 & 23 & 19 & 10 & 12 & 1 & 5 & 1 & 25 & 48.3 & 4.6 \\
\hline MZUCR-13887 & 1 & 141 & 27 & 25 & 23 & 19 & 9 & 11 & $1 \mid$ & 5 & 1 & 31 & 61.5 & 6.1 \\
\hline MZUCR-14058 & 1 & 141 & 26 & 25 & 23 & 19 & 9 & 11 & 1 . & 6 & 1 & 31 & 38 & 4.3 \\
\hline MZUCR-14131 & 1 & 148 & 30 & 25 & 23 & 21 & 10 & 12 & 1 & 6 & 1 & 22 & 60.8 & 6.5 \\
\hline MZUCR-15328 & 1 & 134 & 27 & 23 & 23 & 19 & 9 & 11 & 1 & 4 & 1 & 21 & 46.5 & 6 \\
\hline MZUCR-15329 & 1 & 142 & 29 & 23 & 23 & 19 & 10 & 12 & 1 & 6 & 1 & 18 & 58.6 & 6.6 \\
\hline MZUCR-15330 & 1 & 139 & 26 & 25 & 23 & 19 & 10 & 12 & $1 \mid$ & 5 & 1 & 21 & 58.5 & 5.6 \\
\hline MZUCR-15331 & 1 & 142 & 28 & 25 & 23 & 19 & 10 & 12 & \begin{tabular}{|l|l}
1 \\
\end{tabular} & 6 & 1 & 23 & 62 & 6.1 \\
\hline MZUCR-15332 & 1 & 140 & 29 & 23 & 23 & 19 & 10 & 12 & $1 \mid$ & 5 & 1 & 23 & 52.3 & 5.2 \\
\hline MZUCR-16540 & 1 & 143 & 27 & 23 & 23 & 19 & 10 & 12 & 1 & 5 & 1 & 25 & 61.5 & 7 \\
\hline MZUCR-16803 & 1 & 142 & 33 & 23 & 23 & 19 & 10 & 12 & 1 & 6 & 1 & 29 & 35 & 4.3 \\
\hline MZUCR-17025 & 1 & 139 & 27 & 25 & 23 & 19 & 11 & 13 & 1 . & 5 & 1 & 24 & 46.2 & 5.4 \\
\hline MZUCR-19212 & 1 & 144 & 27 & 23 & 23 & 19 & 10 & 12 & $1 \mid$ & 6 & 1 & 20 & 53.3 & 5.3 \\
\hline MZUCR-19821 & 1 & 141 & 28 & 25 & 23 & 19 & 10 & 12 & $1 \mid$ & 6 & 1 & 20 & \begin{tabular}{|l|}
71.3 \\
\end{tabular} & 8.1 \\
\hline MZUCR-20780 & 1 & 136 & 25 & 23 & 23 & 19 & 9 & 11 & 1 & 6 & 1 & 22 & 22.3 & 2.9 \\
\hline MZUCR-41 & 1 & 141 & 28 & 25 & 23 & 21 & 9 & 12 & \begin{tabular}{|l|l|}
1 \\
\end{tabular} & 5 & 2 & 32 & 44.4 & 4.8 \\
\hline MZUCR-308 & 1 & 137 & 33 & 25 & 23 & 19 & 9 & 11 & \begin{tabular}{|l|l}
1 \\
\end{tabular} & 5 & 2 & 26 & 33.4 & 5 \\
\hline
\end{tabular}




\section{Continue Table 2.}

\begin{tabular}{|c|c|c|c|c|c|c|c|c|c|c|c|c|c|c|}
\hline Museum Catalog & pecies & 1 & 2 & 3 & 4 & 5 & 6 & 7 & 8 & 9 & 10 & 11 & 12 & 13 \\
\hline MZUCR-1431 & 1 & 135 & 31 & 25 & 23 & 19 & 9 & 11 & 1 & 5 & 2 & 19 & 33.6 & 5.5 \\
\hline MZUCR-2722 & 1 & 36 & 29 & 25 & 25 & 21 & 10 & 12 & 1 & 5 & 2 & 19 & 35.1 & 4.5 \\
\hline IZUC & 1 & 37 & 29 & 23 & 23 & 19 & 9 & 11 & 1 & 5 & 2 & 25 & 33.8 & 5.1 \\
\hline MZUCR-2 & 1 & 40 & 29 & 25 & 23 & 19 & 9 & 11 & 1 & 5 & 2 & 23 & 33.4 & 3.5 \\
\hline MZI & 4 & 38 & 34 & 23 & 23 & 19 & 9 & 11 & 1 & 6 & 2 & 27 & 39.5 & 6 \\
\hline MZUC & 1 & 37 & 31 & 23 & 23 & 19 & 10 & 12 & 1 & 5 & 2 & 23 & 19 & 2.2 \\
\hline MZL & 1 & 38 & 27 & 25 & 23 & 19 & 9 & 11 & 1 & 5 & 2 & 28 & 27 & 3.5 \\
\hline MZUCR- & 1 & 38 & 31 & 25 & 25 & 21 & 10 & 12 & 1 & \begin{tabular}{|l|}
3 \\
\end{tabular} & 2 & 23 & 51 & 5.5 \\
\hline $\mathrm{MZU}$ & 7 & 35 & 32 & 25 & 23 & 21 & 10 & 10 & 1 & 6 & 2 & 22 & 37.2 & 5.8 \\
\hline MZUC & 1 & 39 & 32 & 25 & 23 & 19 & 9 & 11 & 1 & 5 & 2 & 22 & 40.1 & 5.8 \\
\hline $\mathrm{MZZ}$ & 1 & 36 & 33 & 25 & 23 & 19 & 9 & 11 & 1 & 5 & 2 & 21 & 44.5 & 6.5 \\
\hline $\mathrm{MZL}$ & 1 & 34 & 28 & 23 & 23 & 19 & 10 & 10 & 1 & 5 & 2 & 24 & 28.1 & 4.4 \\
\hline & 1 & 42 & 30 & 23 & 23 & 19 & 9 & 11 & 1 & 5 & 2 & 21 & 39.8 & 5.9 \\
\hline MZL & 2 & 68 & 35 & 25 & 25 & 19 & 9 & 11 & 2 & 6 & 1 & 28 & 33.8 & 4.6 \\
\hline & 2 & 68 & 39 & 27 & 27 & 21 & 11 & 13 & 2 & 6 & 1 & 28 & 62.6 & 8.1 \\
\hline $\mathrm{MZU}$ & 2 & 63 & 34 & 25 & 25 & 19 & 9 & 11 & 2 & 7 & 1 & 28 & 49.8 & 5.7 \\
\hline $\mathrm{M} 2$ & 2 & 60 & 40 & 25 & 25 & 19 & 10 & 12 & 2 & 5 & 1 & 35 & 36.7 & 5.5 \\
\hline $\mathrm{MZ}$ & 2 & 68 & 33 & 25 & 25 & 19 & 11 & 13 & 2 & 7 & 1 & 30 & 17.5 & 2.5 \\
\hline $\mathrm{MZ}$ & 2 & 76 & 37 & \begin{tabular}{|l|}
21 \\
\end{tabular} & 27 & 19 & 10 & 12 & 2 & 6 & 1 & 28 & 22 & 4 \\
\hline & & & 33 & 25 & 25 & 19 & 1 & 12 & 2 & 5 & 1 & & 1 & 8 \\
\hline MZ & 2 & 72 & 34 & 27 & 27 & 19 & 10 & 12 & 2 & 6 & 1 & 28 & 78.8 & 9.2 \\
\hline & 2 & 73 & 27 & 25 & 25 & 19 & 11 & 11 & 2 & 6 & 1 & 30 & 67.4 & 8 \\
\hline $\mathrm{MZ}$ & 2 & 65 & 35 & 25 & 25 & 19 & 10 & 11 & 2 & 6 & 1 & 29 & 73.3 & 9.1 \\
\hline $\mathrm{MZ}$ & 2 & 75 & 34 & 27 & 27 & 19 & 11 & 12 & 2 & \begin{tabular}{|l|}
7 \\
\end{tabular} & 1 & 28 & 76.1 & 9.1 \\
\hline $\mathrm{MZ}$ & 0 & 67 & 41 & 25 & 25 & 19 & 10 & 12 & 2 & 6 & 1 & 28 & 22 & 3.5 \\
\hline $\mathrm{MZ}$ & 2 & 60 & 29 & 23 & 25 & 19 & 9 & 11 & 2 & 5 & 1 & 29 & 22.4 & 3.2 \\
\hline $\mathrm{Mz}$ & 7 & & 36 & 25 & 27 & 19 & 11 & 13 & 2 & 6 & 1 & 30 & 22.2 & 2.4 \\
\hline $\mathrm{M}_{2}$ & 2 & 76 & 35 & 25 & 25 & 19 & 10 & 10 & 2 & 6 & 1 & 30 & 87.2 & 9.6 \\
\hline & 7 & & 38 & 25 & 25 & 19 & 9 & 11 & 2 & 5 & 1 & 38 & 42 & 6 \\
\hline $\mathrm{M}$ & 2 & 165 & 38 & 25 & 25 & 19 & 10 & 12 & 2 & 6 & 1 & 34 & 75.2 & 8.9 \\
\hline 60 & 2 & 164 & 35 & 23 & 25 & 19 & 9 & 11 & 2 & \begin{tabular}{|l|}
7 \\
\end{tabular} & 1 & 34 & 36.1 & 5.3 \\
\hline $\mathrm{MZL}$ & 2 & 160 & 26 & 25 & 25 & 19 & 10 & 12 & 2 & 5 & 2 & 34 & 22.5 & 3.1 \\
\hline $\mathrm{MZU}$ & 2 & 160 & 38 & 27 & 25 & 21 & 10 & 12 & 2 & 6 & 2 & 36 & 33.5 & 5 \\
\hline $\mathrm{MZU}$ & 2 & 163 & 39 & 25 & 23 & 19 & 10 & 12 & 2 & 6 & 2 & 48 & 47.2 & 6.7 \\
\hline & 2 & 7 & 42 & 25 & 25 & 19 & 11 & 11 & 2 & 5 & 2 & 42 & 18.2 & 7 \\
\hline MZI & 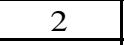 & 166 & 33 & 25 & 25 & 19 & 9 & 11 & 2 & 5 & 2 & 33 & 18.4 & 3.5 \\
\hline MZUCR- & 2 & 166 & 39 & 25 & 25 & 19 & 11 & 13 & 2 & 6 & 2 & 43 & 52.7 & 8.8 \\
\hline MZUCR-8592 & 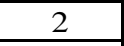 & 164 & 41 & 25 & 25 & 19 & 10 & 12 & 2 & 6 & 2 & 34 & 59.2 & 9 \\
\hline MZUCR-13838 & 2 & 162 & 38 & 25 & 25 & 19 & 10 & 12 & 2 & 6 & 2 & 38 & 33.6 & 5.2 \\
\hline MZUCR-13919 & 2 & 161 & 35 & 25 & 25 & 19 & 10 & 10 & 2 & 5 & 2 & 33 & 17.7 & 3.5 \\
\hline MZUCR-14108 & 2 & 163 & 39 & 25 & 25 & 19 & 9 & 11 & 2 & 5 & 2 & 32 & 58 & 8.5 \\
\hline MZUC & 2 & 164 & 40 & 25 & 25 & 19 & 10 & 12 & 2 & 5 & 2 & 48 & 59 & 9 \\
\hline MZUCR-15287 & 2 & 162 & 39 & 23 & 25 & 19 & 9 & 11 & 2 & \begin{tabular}{|l|l}
5 \\
\end{tabular} & 2 & 36 & 64.6 & 9 \\
\hline
\end{tabular}


Continue Table 2.

\begin{tabular}{|c|c|c|c|c|c|c|c|c|c|c|c|c|c|c|}
\hline \begin{tabular}{|l|} 
Museum Catalog \\
\end{tabular} & \begin{tabular}{|l|} 
Species \\
\end{tabular} & 1 & 2 & 3 & 4 & 5 & 6 & 7 & 8 & 9 & 10 & 11 & 12 & 13 \\
\hline MZUCR-15288 & 2 & 170 & 39 & 25 & 25 & 19 & 10 & 12 & 2 & 5 & 2 & 39 & 65.2 & 9.1 \\
\hline MZUCR-15294 & 2 & 166 & 39 & 25 & 23 & 19 & 10 & 12 & 2 & 6 & 2 & 39 & 68 & 9.5 \\
\hline MZUCR-15296 & 2 & 168 & 44 & 25 & 25 & 19 & 10 & 12 & 2 & 6 & 2 & 31 & 23 & 3.2 \\
\hline MZUCR-15298 & 2 & 153 & 35 & 25 & 25 & 19 & 10 & 12 & 2 & 7 & 1 & 24 & 26.3 & 3.5 \\
\hline MZUCR-15300 & 2 & 145 & 28 & 23 & 25 & 19 & 10 & 10 & 2 & 4 & 2 & 32 & 21.6 & 3.8 \\
\hline MZUCR-15301 & 2 & 167 & 42 & 25 & 25 & 19 & 10 & 12 & 2 & 6 & 2 & 35 & \begin{tabular}{|l|}
75.2 \\
\end{tabular} & 10.1 \\
\hline MZUCR-15302 & 2 & 166 & 37 & 21 & 23 & 19 & 9 & 11 & 2 & 6 & 2 & 27 & 62.5 & 8.6 \\
\hline MZUCR-15476 & 2 & 166 & 37 & 25 & 25 & 19 & 10 & 10 & 2 & 7 & 2 & 33 & \begin{tabular}{|l|}
70.9 \\
\end{tabular} & 7.6 \\
\hline MZUCR-20838 & 2 & 161 & 35 & 25 & 25 & 19 & 9 & 11 & 2 & 5 & 2 & 33 & 45.5 & 6.6 \\
\hline CEREO-109 & 3 & 153 & 26 & 25 & 25 & 19 & 9 & 11 & 1 & 5 & 1 & 18 & 54 & 5 \\
\hline CEREO-115 & 3 & 162 & 32 & 25 & 25 & 19 & 11 & 13 & 1 & 5 & 1 & 19 & 73 & 7 \\
\hline CEREO-120 & 3 & 155 & 28 & 25 & 25 & 19 & 9 & 11 & 1 & 5 & 1 & 19 & 62 & 5 \\
\hline CEREO-213 & 3 & 156 & 23 & 23 & 23 & 19 & 10 & 12 & 1 & 5 & 1 & 23 & 54 & 4.2 \\
\hline CEREO-235 & 3 & 164 & 32 & 23 & 23 & 19 & 9 & 11 & 1 & 5 & 1 & 21 & 52 & 5.5 \\
\hline CEREO-329 & 3 & 154 & 29 & 23 & 25 & 19 & 9 & 11 & 1 & 5 & 1 & 26 & 62 & 5.3 \\
\hline NMNH-129876 & 3 & 165 & 33 & 25 & 25 & 19 & 9 & 11 & 1 & 5 & 1 & 17 & 50.9 & 4.8 \\
\hline NMNH-297735 & 3 & 161 & 32 & 25 & 27 & 21 & 10 & 14 & 1 & 6 & 1 & 20 & 21.5 & 2.2 \\
\hline CAS-21061 & 3 & 154 & 29 & 27 & 27 & 21 & 10 & 12 & 1 & 6 & 1 & 20 & 55.8 & 5.4 \\
\hline \begin{tabular}{|l|} 
CAS-21062 \\
\end{tabular} & 3 & 155 & 31 & 27 & 25 & 19 & 10 & 12 & 1 & 5 & 1 & 16 & \begin{tabular}{|l|}
35.8 \\
\end{tabular} & 3.2 \\
\hline MVZ-78766 & 3 & 153 & 33 & 25 & 25 & 19 & 9 & 11 & 1 & 5 & 1 & 18 & 39.2 & 4.8 \\
\hline FLMNH-39818 & 3 & 156 & 29 & 27 & 27 & 21 & 9 & 12 & 1 & 5 & 1 & 18 & 60.3 & 5.1 \\
\hline FLMNH-127697 & 3 & 159 & 31 & 27 & 25 & 19 & 9 & 12 & 1 & 5 & 1 & 22 & 55.6 & 4.7 \\
\hline FLMNH-74277 & 3 & 154 & 29 & 25 & 25 & 19 & 10 & 12 & 1 & 3 & 1 & 17 & 24.9 & 2.1 \\
\hline KU-112579 & 3 & 159 & 30 & 25 & 25 & 21 & 11 & 14 & 1 & 7 & 1 & 18 & \begin{tabular}{|l|}
23.2 \\
\end{tabular} & 1.6 \\
\hline KU-112580 & 3 & 162 & 33 & 25 & 25 & 19 & 9 & 11 & 1 & 5 & 1 & 24 & 46.3 & 4.7 \\
\hline FMNH-Chi-68051 & 3 & 152 & 32 & 25 & 25 & 19 & 10 & 12 & 1 & 6 & 1 & 17 & 33.4 & 3.3 \\
\hline CEREO-112 & 3 & 147 & 26 & 23 & 23 & 19 & 9 & 11 & 1 & 5 & 2 & 27 & 45.7 & 4.5 \\
\hline CEREO-116 & 3 & 154 & 29 & 25 & 27 & 19 & 10 & 12 & 1 & 5 & 2 & 21 & 51 & 5.5 \\
\hline CEREO-118 & 3 & 158 & 31 & 25 & 25 & 21 & 10 & 12 & 1 & 5 & 2 & 18 & 57 & 5.5 \\
\hline NMNH-140676 & 3 & 154 & 34 & 25 & 25 & 19 & 9 & 12 & 1 & 5 & 2 & 18 & 45.9 & 3.6 \\
\hline CAS-98362 & 3 & 153 & 34 & 25 & 25 & 19 & 9 & 10 & 1 & 6 & 2 & 19 & 25.1 & 2.5 \\
\hline MVZ-83442 & 3 & 157 & 34 & 23 & 25 & 19 & 9 & 11 & 1 & 5 & 2 & 15 & 31.4 & 3.3 \\
\hline FLMNH-39819 & 3 & 155 & 34 & 23 & 23 & 19 & 9 & 11 & 1 & 5 & 2 & 18 & 42.3 & 4.5 \\
\hline \begin{tabular}{|l|} 
KU-112588 \\
\end{tabular} & 3 & 149 & 31 & 25 & 25 & 19 & 9 & 11 & 1 & 5 & 2 & 17 & \begin{tabular}{|l|}
41.6 \\
\end{tabular} & 4.1 \\
\hline KU-112578 & 3 & 158 & 34 & 27 & 25 & 17 & 9 & 12 & 1 & 6 & 2 & 18 & 48.9 & 4.2 \\
\hline KU-112576 & 3 & 153 & 34 & 25 & 25 & 19 & 9 & 10 & 1 & 4 & 2 & 19 & \begin{tabular}{|l|}
38.4 \\
\end{tabular} & 3.9 \\
\hline MZUCR-11643 & 4 & 159 & 31 & 27 & 25 & 19 & 10 & 12 & 1 & 8 & 1 & 27 & \begin{tabular}{|l|}
44.2 \\
\end{tabular} & 4.2 \\
\hline MZUCR-11644 & 4 & 161 & 19 & 27 & 23 & 19 & 9 & 11 & 1 & 6 & 1 & 24 & 45.4 & 3.4 \\
\hline MZUCR-11642 & 4 & 157 & 35 & 27 & 25 & 21 & 9 & 11 & 1 & 7 & 2 & 28 & 25.5 & 2.6 \\
\hline MZUCR-3310 & 5 & 144 & 27 & 25 & 25 & 21 & 10 & 12 & 1 & 5 & 1 & 19 & 29 & 3.6 \\
\hline MZUCR-3359 & 5 & 142 & 30 & 23 & 23 & 19 & 9 & 11 & 1 & 5 & 2 & 22 & 37.3 & 3.9 \\
\hline MZUCR-11479 & 5 & 140 & 31 & 25 & 23 & \begin{tabular}{|l|}
21 \\
\end{tabular} & 10 & \begin{tabular}{|l|}
12 \\
\end{tabular} & 1 & 7 & 2 & 21 & \begin{tabular}{|l|}
20.1 \\
\end{tabular} & 2.1 \\
\hline
\end{tabular}




\section{RESULTS}

Porthidium lansbergii and Porthidium cf lansbergii from Panama show great variation in meristic characters (Table 2). Briefly, ventral scales ranged, including both sexes, from 147 to 165 and caudal scales from 23 to 34 . The mean ( \pm S.E.) number of ventral scales is higher in females $(157.3 \pm 1.0)$ than males $(153.8 \pm 1.1)$, and the differences are statistically significant (Student $t=2.3, d f=25, p=0.03$ ). Conversely, males tend to have a slightly higher mean number of subcaudals ( $32.1 \pm$ $0.8)$ than females $(30.1 \pm 0.6)$, but the differences were marginally significant (Student $t=1.8, d f=25, p=0.08$ ). Males are slightly smaller (mean: $42.7 \pm 2.9 \mathrm{~cm}$ ) than females (mean: $47.2 \pm 3.6 \mathrm{~cm}$ ), with no significant differences in their total length $\left(F_{1,22}=0.99, p=0.33\right)$. Overall, body size did not differ among $P$. lansbergii individuals $\left(F_{2,22}\right.$ $=2.30, p=0.12$ ).

Dorsal patterns of $P$. lansbergii consist mostly of pairs of rectangular blotches that are mostly arranged alternately, at each side of the vertebral line. However, in a few individuals, blotches are placed opposite to each other. Coloration pattern and body hue vary slightly among individuals (Fig. 3), but not clear differences in coloration were observed among individuals from eastern, central, or western Panama. The number of body blotches ranged from 15 to 34 (Table 2), but no differences were observed in this variable between sexes $\left(F_{1,22}=0.09, p=0.76\right)$, or among regions $\left(F_{2,22}=0.96, p=0.39\right)$. Intersupraoculars, canthals, and the number of dorsal scale rows showed the lowest variation in our $P$. lansbergii sample.

A PCA performed over meristic characters allowed partial discrimination among some hog-nosed pit-viper species (Fig. 4A). The first component accounts for $77.9 \%$ of the variance and mainly differentiated three groups: $P$. ophryomegas, $P$. nasutum/P. porrasi, and $P$. lansbergii/P. volcanicum. This component is correlated with ventral scale count $(\mathrm{r}=0.92)$, and less strongly with number of subcaudals $(\mathrm{r}=$ $0.24)$, as well as the number of blotches $(r=0.28)$. The second component is accounts for $15.9 \%$ of the variation and is mainly explained by the number of blotches $(\mathrm{r}=0.91)$, separating $P$. lansbergii from other species. No discrimination between $P$ nasutum and $P$. porrasi 
was possible from this data set; and $P$. volcanicum shares characteristics with $P$. ophrymegas and $P$. lansbergii (Fig. 4A). Our PCA results for $P$. lansbergii from Panama (Fig. 4B) accounted for $77 \%$ of the total variance with the first two components, suggesting a relatively high morphological overlapping among specimens from the three regions studied.
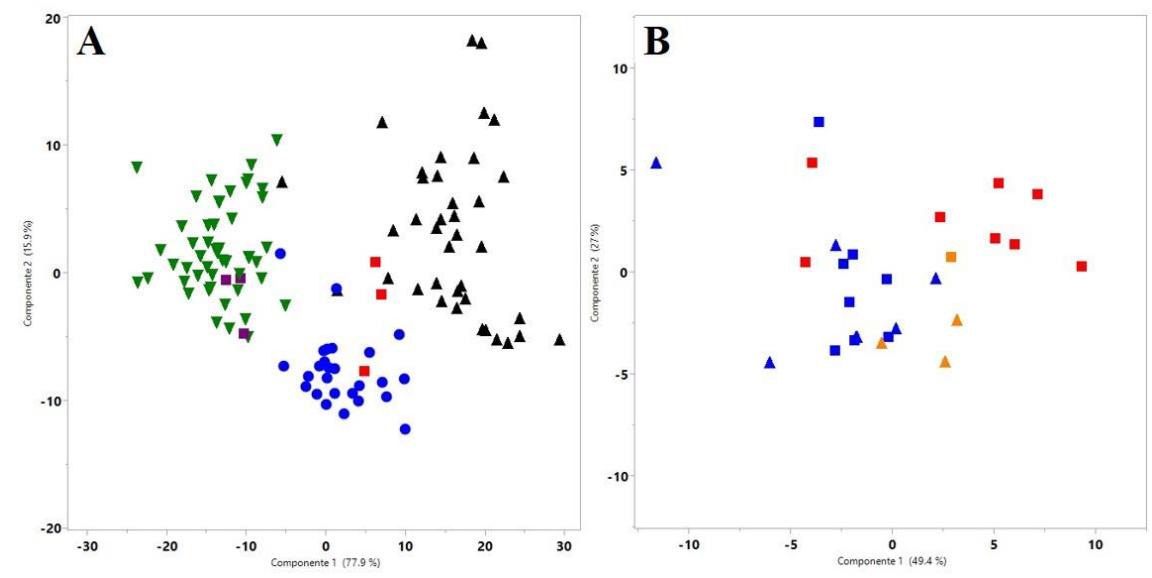

Fig. 4 Principal Component Analysis with Manhattan distance for all species in this study (A) (dark blue $=P$. lansbergii; green $=P$. nasutum; purple $=P$. porrasi; black $=P$. ophryomegas; and red $=P$. volcanicum $)$ and (B) only for P. lansbergii specimens from Panama (Females=triangle, Males=squares; red=western, blue=central, orange $=$ eastern Panama)

Our phylogenetic analyses recovered a partial phylogeny of Porthidium that is consistent with previous reconstructions of hog-nosed pit-viper phylogenies (Fig. 5). Briefly, an early divergence produces two main clusters, one including $P$. dunni and $P$. ophryomegas, and the other includes $P$. yucatanicum as the sister taxon of a clade including $P$. nasutum/P. porrasi and $P$. lansbergii. Further, $P$. lansbergii comprises a monophyletic group that includes both western, central and eastern Panama specimens, as well as the individual from Venezuela. Therefore, the enigmatic western Panama specimens are identified as members of this species. 


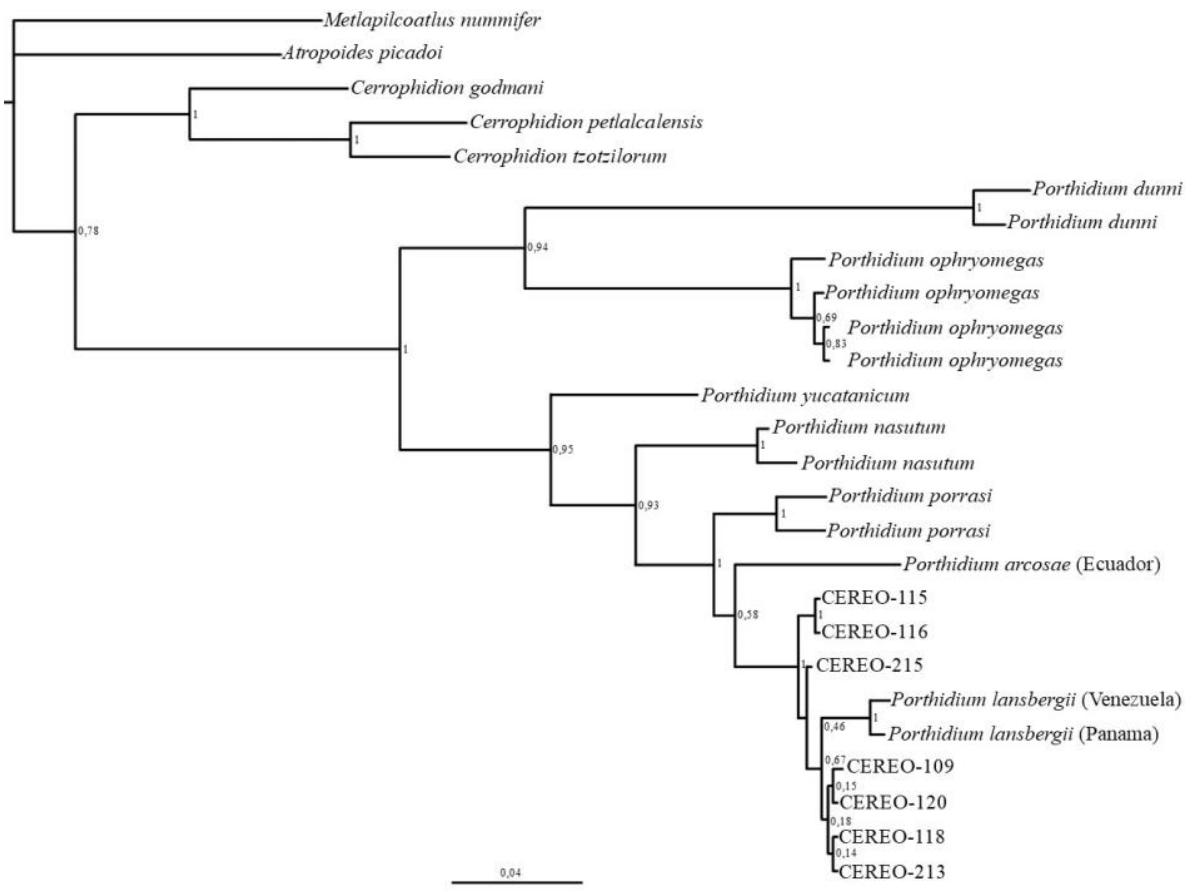

Fig. 5 Phylogram Phylogenetic tree constructed based on Bayesian inference

\section{DISCUSSION AND CONCLUSIONS}

Campbell and Lamar (1989, 2004), and Kohler (2003) provided several records of $P$. lansbergii in Panama as part of their claim that it was restricted to the central and eastern half of the country. Ray and Knight (2013) considered the distribution of P. lansbergii throughout the Pacific slope of Panama, but localities or records were not given. Our extensive literature search recovered records, omitted in previous reviews, of this species from the western Pacific slope of Panama. For instance, Dunn (1949) mentioned specimens described as Bothrops (=Porthidium) lansbergii collected in localities within the Cocle-Herrera region. Recently, the species was reported by Martínez-Cortés et al (2005) in 'El Montuoso' Forest Reserve in the Herrera province and by MontezaMoreno, C. M. (pers. obs., 2017) in the locality of Mariato, Veraguas Province. Furthermore, museum records and new collections of 
individuals show that this species inhabit in the provinces of Chiriqui and Veraguas. The distribution of the nominal P. lansbergii in Lower Central America extends to the western Pacific of Panama (Fig. 1). Thus, since our records lie near the border of Costa Rica, the species could potentially occur there.

Despite putative differences in coloration patterns, we cannot distinguish enigmatic individuals from those identified as $P$. lansbergii from central and eastern Panama based in scutellation. In addition, our phylogenetic analysis clearly supports the monophyly of $P$. lansbergii from Panama, and does not provide any evidence for the existence of multiple lineages within the nominal species in this country. However, our dataset does not include specimens from the entire distribution range of $P$. lansbergii and the fact that the species shifts its distribution from the Pacific slopes in Panama to the Atlantic in Colombia up to Venezuela is interesting and warrants further scrutiny.

Two other members of Porthidium are also know to occur in Panama: $P$. nasutum, and $P$. volcanicum. The first one inhabits the humid environments along the Caribbean lowlands and piedmonts, crossing to the Pacific side in Darien; therefore, it is allopatric to P. lansbergii populations. Porthidium. volcanicum, a species ostensibly endemic to a small area in Costa Rica, was reported in Boquete, Chiriquí, in western Panama (Dwyer and Van den Burgh, 2012). It is likely that this species can be sympatric with $P$. lansbergii; however, further studies are required to depict distribution ranges for both species.

Our data showed that ventral scale counts and number of blotches are sufficient to distinguish among hog-nosed pit-vipers' species. Differences in ventral scales, and subtle color variation, were used to distinguish three subspecies of Porthidium lansbergii (Roze, 1959; Peters, 1968; Sandner-Montilla, 1989; Fuentes and Rodriguez 1997). However, our data suggest a great variation in meristic characters, including a higher ventral scale range than considered in these studies. Additional studies are highly recommended for a comprehensive understanding of the variability in morphological traits across the species geographic distribution. Moreover, since the species occurs in 
seasonally-dry and humid environments along its distribution, it is possible that variation in scutellation characters results from environmentally driven effects, as it has been observed in other wide ranged snakes. For instance, Saldarriaga-Córdoba et al. (2009) found that geographic variation of dorsal blotches, ventral scales and other meristic characters in Bothrops asper correlates to external factors, such as latitude, the number of dry months, and precipitation. P. lansbergii, as well as $P$. nasutum which also shifts its distribution from the Atlantic into the Pacific in eastern Panama down to Ecuador, provides an ideal opportunity to understand morphometric responses to environmental variables.

\section{ACKNOWLEDGMENT}

We thank Nimiadina Herrera, Oris Sanjur, and Ángel Aguirre for logistic support in the Smithsonian Tropical Research Institute molecular lab and library; Criselda Castillo and Alonso Santos from the University of Panama for helping with technical support and photographing the specimens; Fabián Bonilla for logistic support during C.M.M-M. visits to Costa Rica and Monica Saldarriaga for helping with the Bayesian phylogenetic analysis. We are grateful to the University of Panama, Smithsonian Tropical Research Institute, and especially Anthony Coates for the funding provided for this research, including research supplies and visits to the National Museum of Natural History in Washington DC, American Museum of Natural History in New York, and the 'Museo de Zoologia' of the University of Costa Rica in San Jose. We also like to acknowledge each of the institutions/museums, the curators, and especially to the Herpetology Division at the Museum Support Center of the NMNH for all support provided with the logistic to borrow specimens from the different museums and during C.M.M-M visits to the museum. This project was approved by the Vice-chancellor of Research and Grad Studies of the University of Panama, with registration code: VIP-01-04-00-05-2011-13.

\section{REFERENCES}

Campbell, J. A. \& W. W. Lamar. 1989. The venomous reptiles of Latin 
America. Cornell University Press, Ithaca, New York, United States. Campbell, J. A. \& W. W. Lamar. 2004. The venomous reptiles of the western hemisphere. Cornell University Press, Ithaca, New York, United States.

Campbell, J. A., D. R. Frost, \& T. A. Castoe. 2019. New generic name for jumping pitvipers (Serpentes: Viperidae). Revista Latinoamericana de Herpetologia 2: 52:53.

Castoe, T., M. Sasa, \& C. L. Parkinson. 2005. Modeling nucleotide evolution at the mesoscale: The phylogeny of the Neotropical pitvipers of the Porthidium group (Viperidae: Crotalinae). Molecular Phylogenetics and Evolution 37: 881-898.

Chippindale, P.T., A. H. Price, J. J. Wiens, \& D. M. Hillis. 2000. Phylogenetic relationships and systematics revision of central Texas hemidactyliine plethodontid salamanders. Herpetological Monographs 14: $1-80$.

Cope, E. D. 1871. Ninth contribution to the herpetology of tropical America. Proceedings of the Academy of Natural Sciences Philadelphia, 23: $200-224$.

Dunn, E. R. 1949. Relative abundance of some Panamanian snakes. Ecology 30(1): 39-57.

Dwyer. Q., \& J. Van Den Burgh. 2012. Geographic distribution: Serpentes: Porthidium volcanicum. Herpetological Review 43(2): 308

Fuentes, O., \& A. Rodríguez-Acosta. 1997. On the genera Bothriechis, Bothriopsis, Bothrops and Porthidium (Serpentes: Crotalidae) in Venezuela. Keys of biomedical interest for their identification. Acta Biologica Venezuelica 17: 31-38.

$\mathrm{JMP}^{\circledR}$, Version 13, SAS Institute Inc., Cary, NC, 1989-2019.

Köhler, G. 2003. Reptiles of Central America. Herpeton, Offenbach, Germany. 
Palumbi, S. R. 1996. Nucleic acids II: The polymerase chain reaction. Pp. 205-247. In D.M. Hillis, C. Moritz, and B. K. Mable, Molecular Systematic. Sinauer Association, Sunderland, Massachusetts, United States.

Porras, L., J. R. McCranie, \& L. D. Wilson. 1981. The systematics and distribution of the hognose viper Bothrops nasuta Bocourt (Serpentes: Viperidae). Tulane Stud. Zool. Bot. 22: 85-107.

Qiagen, A. G. 2006. DNeasyC blood and tissue handbook. Qiagen AG, Hombrechtikon, Switzerland.

Ray, J. M. \& Knight, J. L. 2013. The venomous snakes and their mimics of Panama and Costa Rica: Las culebras venenosas y sus mimicas de Panama y Costa Rica. Team Snake Panama.

Martínez-Cortés, V., A. Rodríguez, \& C. Garibaldi. 2005. Inventario de reptiles en los bosques secundarios de la reserva forestal El Montuoso, Provincia de Herrera, Panama. In: Garibaldi, C. (eds) Diversidad biologica y servicios ambientales de los fragmentos de bosque en la reserve forestal El Montuoso, Panama, pp 119 - 127

Roze, J., 1959. Taxonomic Notes on a collection of Venezuelan reptiles in the American Museum of Natural History. American Museum Novivates 1934: 1-14.

Saldarriaga-Córdoba, M. M., M. Sasa, R. Pardo, \& M. A. Méndez. 2009. Phenotypic differences in a cryptic predator: Factors influencing morphological variation in the terciopelo Bothrops asper (Garman, 1884; Serpentes: Viperidae). Toxicon 54(7): 923-937.

Sandner-Montilla, F. 1989. Una nueva especie de Bothrops lansbergii de la familia Crotalidae. Memorias Científicas de Ofidiología 9: 1-16.

Savage, J. M., 2002. The amphibians and reptiles of Costa Rica: a herpetofauna between two continents, between two seas. University of Chicago press.

Recibido 6 de enero de 2020, aceptado 13 de febrero de 2020. 